\title{
Infections in patients undergoing craniotomy: risk factors associated with post-craniotomy meningitis
}

\author{
*Irene S. Kourbeti, MD, PhD, ${ }^{1}$ Antonis F. Vakis, MD, PhD, ${ }^{2}$ Panayiotis Ziakas, MD, PhD, ${ }^{3}$ \\ Dimitris Karabetsos, MD, ${ }^{2}$ Evangelos Potolidis, MD, ${ }^{4}$ Silvana Christou, MD, ${ }^{5}$ \\ and George Samonis, MD, $\mathrm{PhD}^{6}$
}

\begin{abstract}
${ }^{1}$ Department of Internal Medicine, General Hospital of Chalkida; Departments of ${ }^{2}$ Neurosurgery and ${ }^{6}$ Department of Internal Medicine/Infectious Disease, University Hospital of Heraklion; ${ }^{4}$ Department of Internal Medicine, General Hospital of Volos, Greece; ${ }^{3}$ Department of Internal Medicine, Division of Infectious Disease, Warren Alpert Medical School, Brown University, Providence, Rhode Island; and ${ }^{5}$ Department of Internal Medicine, General Hospital of Nicosia, Cyprus
\end{abstract}

\begin{abstract}
OBJECT The authors performed a prospective study to define the prevalence and microbiological characteristics of infections in patients undergoing craniotomy and to clarify the risk factors for post-craniotomy meningitis.

METHODS Patients older than 18 years who underwent nonstereotactic craniotomies between January 2006 and December 2008 were included. Demographic, clinical, laboratory, and microbiological data were systemically recorded. Patient characteristics, craniotomy type, and pre- and postoperative variables were evaluated as risk factors for meningitis

RESULTS Three hundred thirty-four procedures were analyzed (65.6\% involving male patients). Traumatic brain injury was the most common reason for craniotomy. Almost $40 \%$ of the patients developed at least 1 infection. Ventilatorassociated pneumonia (VAP) was the most common infection recorded (22.5\%) and Acinetobacter spp. were isolated in $44 \%$ of the cases. Meningitis was encountered in 16 procedures (4.8\%), and CSF cultures were positive for microbial growth in $100 \%$ of these cases. Gram-negative pathogens (Acinetobacter spp., Klebsiella spp., Pseudomonas aeruginosa, Enterobacter cloaceae, Proteus mirabilis) represented $88 \%$ of the pathogens. Acinetobacter and Klebsiella spp. demonstrated a high percentage of resistance in several antibiotic classes. In multivariate analysis, the risk for meningitis was independently associated with perioperative steroid use (OR 11.55, $p=0.005)$, CSF leak (OR 48.03, $p<0.001)$, and ventricular drainage (OR 70.52, $p<0.001)$.

CONCLUSIONS Device-related postoperative communication between the CSF and the environment, CSF leak, and perioperative steroid use were defined as risk factors for meningitis in this study. Ventilator-associated pneumonia was the most common infection overall. The offending pathogens presented a high level of resistance to several antibiotics. http://thejns.org/doi/abs/10.3171/2014.8.JNS132557
\end{abstract}

KEY WORDS craniotomy; meningitis; infection; ventilator-associated pneumonia; surgical site infections

$\mathrm{N}$ OSOCOMIAL meningitis is mainly seen in neurosurgical patients. This complication may result in severe morbidity, with a prolonged length of stay, multiple surgeries, and higher hospital costs. ${ }^{12,27}$ Patients who undergo neurosurgery are also prone to the development of a variety of infections that may pose significant problems for them..$^{14}$

Comprehensive studies have been published on the risk factors for post-craniotomy meningitis (PCM). ${ }^{12,14,24,28} \mathrm{In}$ this study we publish the prospective 3-year experience from the University of Crete on the risk factors associated with PCM. We also analyzed the infections in patients undergoing craniotomies in terms of the prevalence, microbiology, and sensitivities of the offending pathogens.

\section{Methods}

\section{Identification of Patients}

This study took place at the University of Crete. Patients were eligible if they were at least 18 years of age, underwent elective or emergency craniotomy between 2006 and 2008, and survived at least 7 days after surgery.

ABBREVIATIONS CAB/BSI = catheter-associated bacteremia/blood stream infection; EVD = external ventricular drain; ICP = intracranial pressure; ICU = intensive care unit; LOS = length of stay; PCM = post-craniotomy meningitis; SSI = surgical site infection; UTI = urinary tract infection; VAP = ventilator-associated pneumonia.

SUBMITTED November 19, 2013. ACCEPTED August 26, 2014

INCLUDE WHEN CITING Published online October 24, 2014; DOI: 10.3171/2014.8.JNS132557.

DISCLOSURE Dr. Samonis reports receiving non-study related clinical or research support from Pfizer, Novartis, Gilead, and MSD.

* Drs. Kourbeti and Vakis contributed equally to this work. 
Major craniotomies were included. Patients having only CSF shunt or external ventricular drain (EVD) implantations, bur hole trepanation, or stereotactic surgery were excluded. The patients were prospectively followed for the development of meningitis during the first 30 postoperative days and during the 1st postoperative year if a foreign body was implanted. It was assumed that the occurrence of meningitis would result in readmission; although it is possible that admission to another institution may have resulted in some missed cases, it is highly unlikely.

\section{Data Abstraction}

Data were abstracted from the medical charts to a standard database. We recorded comorbidities such as diabetes, malignancy, atherosclerotic cardiovascular disease, chronic renal failure, chronic obstructive pulmonary disease, and perioperative steroid use; prophylactic antibiotic use; characteristics of the procedure performed, such as procedure urgency (elective or emergent), surgery duration, multiple procedures performed at the same time (simultaneous orthopedic or abdominal surgery or facial reconstruction); and the presence of any postoperative CSF drainage, reoperations, or CSF leaks. An operation was considered a revision surgery whenever it was performed through the same incision as a previous operation, regardless of the interval between the 2 procedures. Postoperative CSF leak was recorded when CSF drainage was diagnosed after surgery on the basis of otorrhea, rhinorrhea, or leakage from the surgical wound.

Meningitis was diagnosed according to the definitions of the Centers for Disease Control. ${ }^{8}$ The definition of the disease was as follows: 1) organisms cultured from CSF, 2) at least 1 of the following signs or symptoms with no other recognized cause: fever $\left(>38^{\circ} \mathrm{C}\right)$, headache, stiff neck, meningeal signs, cranial nerve signs, or irritability and if diagnosis was made antemortem, attending physician instituted appropriate antimicrobial therapy, and at least one of the following: a) increased WBC count, elevated protein level, and/or decreased glucose level in CSF; b) organisms seen on gram stain of CSF; c) organisms cultured from blood; d) positive antigen test of CSF, blood, or urine; e) diagnostic single antibody titer (IgM) or 4-fold increase in paired sera (IgG) for pathogen. We recorded the postoperative day on which the diagnostic lumbar puncture was performed, the organisms identified by CSF culture, and all the infections (ventilator-associated pneumonia [VAP], urinary tract infection [UTI], pneumonia, bacteremia) that developed in this cohort. The data were transferred to a database and were analyzed using SPSS software (Version 16.0, SPSS Inc.). Continuous variables were compared using the Student t-test or the Mann-Whitney U-test, whereas categorical variables were compared using the Fisher exact test. In univariate analysis, odds ratios and $95 \%$ confidence intervals were calculated using the Mantel-Haenszel test. Stepwise multivariate logistic regression was used to model the interactions of those variables significantly associated with surgical site infections (SSIs) or meningitis in univariate analyses. A backward elimination model was used with $\mathrm{P}_{\text {enter }}=0.20$ and $\mathrm{P}_{\text {leave }}=0.05$ to identify independent predictors for meningitis. When a continuous variable such as the number of days of ventricular drainage, was shown to be a statistically significant risk factor, the relationship was modeled further using suitably coded dummy variables. Power calculations were performed using the PASS software (PASS 2008, https://www.ncss.com).

\section{Results}

\section{Study Population and Procedure Characteristics}

The study included 334 procedures in 239 patients who met the inclusion criteria; $65.6 \%$ of the procedures were performed in men. Meningitis developed after 16 (4.8\%) of the procedures. The median age was 48 years for the patients who developed meningitis (interquartile range [IQR] 22-58 years) and 51 (IQR 27-67 years) for those who did not. Traumatic brain injury was the most common reason for craniotomy (accounting for $49.7 \%$ of the procedures). The rest of the craniotomy indications included space-occupying lesions (in $34 \%$ of cases), vascular conditions (10\%), abscess (1\%), and a variety of other reasons (3\%); $18.6 \%$ of the procedures were revision surgeries, and $49.7 \%$ were emergent procedures. Only 3.3\% of the patients had concurrent procedures. Almost $40 \%$ of the patients who underwent craniotomy developed at least 1 infection. SSIs developed after 9\% of the procedures. The overall mortality rate was $15 \%$. There was no statistically significant difference in mortality rate between patients who did and did not develop meningitis.

The median time from admission to surgery was 3 days. The median duration of surgery was 2 hours (IQR 2.0-4.0 hours) for the procedures after which meningitis did not develop and 4 hours (IQR 3.0-6.0 hours) for those after which it did. The median length of stay (LOS) was 27 days (IQR 12-79). Patients who developed meningitis had a much longer LOS (median 191 vs 24.5 days, $\mathrm{p}<$ 0.001). Patients who developed meningitis had a greater propensity to be admitted to the intensive care unit (ICU) ( $p<0.001)$ and a prolonged intubation beyond the time of surgery $(\mathrm{p}<0.001)$.

\section{Meningitis Risk}

The median time from surgery to lumbar puncture was 6.5 days in those cases in which meningitis was diagnosed. Table 1 lists all variables that were examined for their contribution to the risk of meningitis. Individual variables that were statistically significant at a $p$ value less than 0.05 included female sex, perioperative steroid use, increased duration of surgery, presence of CSF leak, ICU admission, use of a central line, and use of ventricular drains. The presence of drains overall was not associated with the development of meningitis. The presence of another SSI was associated with meningitis risk ( $p<$ 0.001). Among the patients who had an SSI other than meningitis, $41 \%$ also developed meningitis. Emergency status of the procedure (i.e., emergent or not) was not associated with the development of meningitis. "Time to surgery" and the presence of skull fractures also were not associated with the development of meningitis. Overall, 14 patients (4\%) had open wound trauma. Patients with open wound trauma were more likely to develop an SSI than those without (36\% vs $11 \%, \mathrm{p}=0.02)$. Also, openwound trauma patients had a higher risk for meningitis 
TABLE 1. Comparison of the 2 groups regarding the development of meningitis

\begin{tabular}{|c|c|c|c|}
\hline Variable & Meningitis & No Meningitis & $p$ Value \\
\hline No. of procedures & 16 & 318 & \\
\hline Female sex & $10(59 \%)$ & $105(33 \%)$ & 0.04 \\
\hline Age (yrs) & & & 0.14 \\
\hline Median & 48 & 51 & \\
\hline IQR & $22-58$ & $27-67$ & \\
\hline LOS (days) & & & $<0.001$ \\
\hline Median & 191 & 24.5 & \\
\hline IQR & $120-259$ & $12-70$ & \\
\hline Diabetes & $1(6 \%)$ & $23(7 \%)$ & 1.0 \\
\hline Malignancy & $2(12 \%)$ & $55(17 \%)$ & 0.74 \\
\hline ASCVD & $4(24 \%)$ & $94(30 \%)$ & 0.79 \\
\hline CRF & $0(0 \%)$ & $9(3 \%)$ & 1.0 \\
\hline COPD & $0(0 \%)$ & $5(2 \%)$ & 1.0 \\
\hline Steroid use & $9(53 \%)$ & $75(24 \%)$ & 0.02 \\
\hline Emergency & $8(48 \%)$ & $158(50 \%)$ & 1.00 \\
\hline Duration in hrs & & & 0.003 \\
\hline Median & 4 & 2 & \\
\hline IQR & $3-6$ & $2-4$ & \\
\hline CSF leak & $6(35 \%)$ & $21(7 \%)$ & 0.001 \\
\hline Multiple procedures & $0(0 \%)$ & $11(3 \%)$ & 1.00 \\
\hline Revision & $2(12 \%)$ & $60(19 \%)$ & 0.75 \\
\hline Entered sinus & $0(0 \%)$ & $23(8 \%)$ & 0.23 \\
\hline Open wound & $2(12 \%)$ & $12(4 \%)$ & 0.16 \\
\hline Dural substitute & $10(59 \%)$ & $156(49 \%)$ & 0.44 \\
\hline ICU admission & $16(100 \%)$ & $170(54 \%)$ & $<0.001$ \\
\hline Intubation & $15(88 \%)$ & $127(40 \%)$ & $<0.001$ \\
\hline TPN & $2(12 \%)$ & $26(8 \%)$ & 0.64 \\
\hline Central line & $15(88 \%)$ & $154(49 \%)$ & $<0.002$ \\
\hline Drain & $16(94 \%)$ & $273(86 \%)$ & 0.49 \\
\hline Ventricular drains & $11(65 \%)$ & $16(5 \%)$ & $<0.001$ \\
\hline ICP placement & $5(29 \%)$ & $88(28 \%)$ & 0.88 \\
\hline Prophylactic antibiotics & $16(94 \%)$ & $324(97 \%)$ & 0.41 \\
\hline
\end{tabular}

ASCVD = atherosclerotic cardiovascular disease $;$ COPD = chronic obstructive pulmonary disease; $\mathrm{CRF}=$ chronic renal failure; $\mathrm{TPN}=$ total parenteral nutrition.

(14\% vs 5\%), but this association failed to reach statistical significance $(p=0.16)$. Crossing a sinus during a procedure was not associated with the development of meningitis $(\mathrm{p}=0.23)$.

Variables that were statistically significant at $p \leq 0.20$ in the univariate analysis were included in a multivariate regression (Table 2). Presence of ventricular drainage (OR 70.52), CSF leak (OR 48.03), and perioperative steroid use (OR 11.55) remained in the model as independent predictors with $\mathrm{p}<0.05$.

\section{CSF Features in Patients With Meningitis}

Postoperative meningitis developed in 16 cases. The characteristics of the CSF in these cases are shown in Table 3.
TABLE 2. Multivariate analysis for the risk for meningitis

\begin{tabular}{lcc}
\hline \multicolumn{1}{c}{ Covariate } & OR $(95 \% \mathrm{Cl})$ & p Value \\
\hline Sex (F vs M) & $2.70(0.68-10.79)$ & 0.16 \\
\hline Steroids (yes vs no) & $11.55(2.08-64.13)$ & 0.005 \\
\hline Duration of surgery & $1.03(0.68-1.55)$ & 0.89 \\
\hline CSF leak (yes vs no) & $48.03(6.75-341.8)$ & $<0.001$ \\
\hline Intubation (yes vs no) & $9.35(0.78-111.7)$ & 0.08 \\
\hline Central line & $0.36(0.02-4.44)$ & 0.42 \\
\hline EVD (yes vs no) & $70.52(10.83-459.1)$ & $<0.001$ \\
\hline
\end{tabular}

\section{Microbiology of Meningitis}

Meningitis was documented by positive CSF culture in all 16 cases. Gram-negative organisms predominated. Acinetobacter spp. were the main isolates (present in $45 \%$ of cases). The other pathogens included: Klebsiella spp. (4 cases), Pseudomonas aeruginosa (2 cases), Enterobacter cloaceae (1 case), Proteus mirabilis (1 case), coagulasenegative staphylococci (1 case), Staphylococcus aureus (1 case), and Candida albicans (1 case). Acinetobacter spp. were fully resistant to $\beta$-lactams, aztreonam, aminoglycosides, and quinolones but they were fully sensitive to colistin. Klebsiella spp. were $100 \%$ resistant to $\beta$-lactams, cephalosporins, aztreonam, and quinolones. Pseudomonas aeruginosa isolates were fully resistant to the carbapenems, but they retained full susceptibility to ticarcillin/clavulanate, piperacillin/tazobactam, cefepime, aztreonam, amikacin, gentamicin, colistin, and ciprofloxacin (Table 4).

\section{Infections Other Than Meningitis}

Ventilator-associated pneumonia was the most common infection in this population $(22.5 \%)$ with $9 \%$ of patients developing an SSI other than meningitis, 9\% a UTI, and $15 \%$ pneumonia not associated with mechanical ventilation. Among SSIs, wound infections predominated (75\%). Postsurgical subdural and epidural abscesses represented only $11 \%$ of the SSIs. Ventriculostomy site, bone cement and bone infections, and subdural empyemas were rare, representing a total of $12 \%$ of the SSIs.

When the meningitis pathogens were compared with the catheter-associated bacteremia/blood stream infection $(\mathrm{CAB} / \mathrm{BSI})$ isolates, in 3 cases the same pathogens grew from the CSF and the blood (namely E. cloacae, Acinetobacter baumannii, and Klebsiella pneumoniae).

\section{Microbiology of Other Infections}

In VAPs, Acinetobacter spp. were the main pathogens, isolated in $44 \%$ of the cases. Pseudomonas aeruginosa

TABLE 3. CSF characteristics of the 16 patients who had meningitis

\begin{tabular}{lcc}
\hline \multicolumn{1}{c}{ Variable } & Median & IQR \\
\hline Protein $(\mathrm{mg} / \mathrm{dl})$ & 120 & $91-634$ \\
\hline Glucose $(\mathrm{mg} / \mathrm{dl})$ & 47 & $40-59$ \\
\hline Glucose CSF/serum ratio & 0.40 & $0.40-0.47$ \\
\hline WBC (cells/ml) & 300 & $220-560$ \\
\hline
\end{tabular}


TABLE 4. Antibiotic resistance in pathogens isolated from meningitis and VAP*

\begin{tabular}{|c|c|c|c|c|c|c|}
\hline \multirow[b]{2}{*}{ Antibiotic } & \multicolumn{3}{|c|}{ Meningitis } & \multicolumn{3}{|c|}{ VAP } \\
\hline & Acinetobacter spp. & Klebsiella spp. & Pseudomonas spp. & Acinetobacter spp. & Klebsiella spp. & Pseudomonas spp. \\
\hline Cephalothin & $100 \%$ & & & $100 \%$ & $67 \%$ & \\
\hline Cefuroxime & $100 \%$ & $100 \%$ & & $100 \%$ & $67 \%$ & \\
\hline Ceftriaxone & $100 \%$ & $100 \%$ & $100 \%$ & $100 \%$ & $67 \%$ & \\
\hline Cefepime & $100 \%$ & $100 \%$ & $0 \%$ & $100 \%$ & $67 \%$ & $37 \%$ \\
\hline Tetracycline & $100 \%$ & $0 \%$ & & $76 \%$ & $22 \%$ & \\
\hline Colistin & $0 \%$ & $20 \%$ & $0 \%$ & $0 \%$ & $33 \%$ & $0 \%$ \\
\hline Ticarcillin/clavunate & $100 \%$ & $100 \%$ & $0 \%$ & $100 \%$ & $67 \%$ & $42 \%$ \\
\hline Piperacillin/tazobactam & $100 \%$ & $100 \%$ & $0 \%$ & $85 \%$ & $67 \%$ & $37 \%$ \\
\hline Imipenem & $33 \%$ & $60 \%$ & $100 \%$ & $67 \%$ & $33 \%$ & $21 \%$ \\
\hline Meropenem & $17 \%$ & $40 \%$ & $100 \%$ & $18 \%$ & $33 \%$ & $26 \%$ \\
\hline Aztreonam & $100 \%$ & $100 \%$ & $0 \%$ & $100 \%$ & $67 \%$ & $37 \%$ \\
\hline Amikacin & $100 \%$ & $80 \%$ & $0 \%$ & $79 \%$ & $33 \%$ & $16 \%$ \\
\hline Gentamicin & $100 \%$ & $80 \%$ & $0 \%$ & $61 \%$ & $33 \%$ & $5 \%$ \\
\hline TMP-SMZ & $100 \%$ & $100 \%$ & $100 \%$ & $82 \%$ & $67 \%$ & $100 \%$ \\
\hline Ciprofloxacin & $100 \%$ & $100 \%$ & $0 \%$ & $100 \%$ & $78 \%$ & $21 \%$ \\
\hline Moxifloxacin & $100 \%$ & $100 \%$ & & $79 \%$ & $67 \%$ & \\
\hline
\end{tabular}

* The percentage values given indicate the percentage of resistance to the antibiotic listed.

was the second most prevalent isolate (25\%). The resistance patterns are shown in Table 4. Of note, the Acinetobacter spp. retained full susceptibility to colistin. The resistance to imipenem approached $67 \%$. Pseudomonas aeruginosa isolates were also fully susceptible to colistin. In UTIs, Klebsiella spp. were the most prevalent pathogens (31\%). The isolates were $100 \%$ resistant to $\beta$ lactams, aztreonam, and quinolones and $75 \%$ resistant to imipenem and colistin.

In SSIs, Acinetobacter spp. predominated, with Klebsiella spp. being the second in prevalence. Gram-positive pathogens were the third most common category. In $\mathrm{CAB} /$ BSI, coagulase-negative staphylococci were the most prevalent pathogens. Gram-positive organisms generally predominated in these infections. Among the gram-negative organisms, A. baumanii was the most common.

\section{Survival Analysis}

After a cumulative follow up of 18,795 days (median 27 days, range 1-318 days), 50 patients had died (15\%)among them, 5 (29\%) of the 16 patients who had meningitis, $1(4 \%)$ of the 23 who had an SSI other than meningitis, 24 (24\%) of the 98 who had other nosocomial infections, and $20(10 \%)$ of the 194 who had no infection. Estimated survival rates at Day 30 were 0.94 (95\% CI 0.65-0.99) for patients with meningitis, 1.0 for patients with other SSIs, 0.84 (95\% CI $0.74-0.90)$ for patients with other nosocomial infections, and 0.90 (95\% CI 0.84-0.94) for those without infections. The corresponding age- and sex-adjusted hazard ratios for patients with infection compared with those without infection were 0.80 (95\% CI $0.22-$ 2.84) for meningitis, 0.38 (95\% CI $0.05-2.89)$ for other SSIs, and 1.43 (95\% CI 0.77-2.63) for other nosocomial infections. The results of all between-group comparisons were also insignificant. Only age predicted an adverse outcome (HR for death 1.03 [95\% CI 1.02-1.05] per year increase in age). In conclusion, there was no statistically significance difference between the study groups with respect to mortality rates (Fig. 1, Table 5).

\section{Discussion}

\section{Prevalence and Microbiology of Post-Craniotomy Meningitis}

The incidence of meningitis in this study was $4.8 \%$, higher than incidences noted in some series ${ }^{2,12,13}$ but not in all. Reichert et al. ${ }^{28}$ in their prospective study reported a rate of $8.9 \%$. In a recent retrospective study from the US, the incidence of post-craniotomy meningitis (PCM) was 5.5\%. ${ }^{14}$ Gram-negative organisms predominated as meningitis pathogens in this prospective cohort. In studies published after 1993, Enterobacteriaceae and other gramnegative rods played a major role. ${ }^{1,6,9,18,28}$ Our results seem to reflect this trend. In this cohort there were no culture-

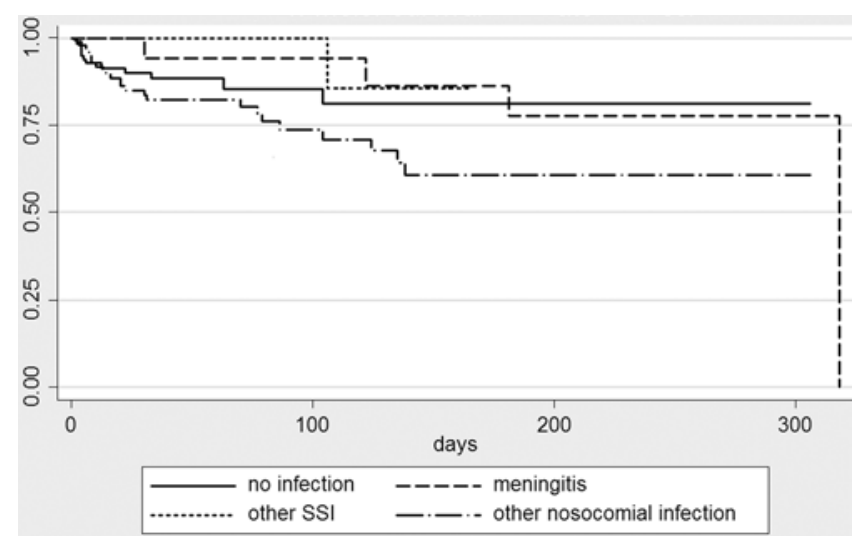

FIG. 1. Kaplan-Meier survival estimates for the patients undergoing craniotomy. 
TABLE 5. Cox regression analysis for mortality according to the type of infections (age-sex adjusted)

\begin{tabular}{lccc}
\hline \multicolumn{1}{c}{ Variable } & $\mathrm{HR}$ & $95 \% \mathrm{Cl}$ & $\mathrm{p}$ Value \\
\hline Age (per yr increase) & 1.03 & $1.02-1.05$ & $<0.001$ \\
\hline Sex & 0.78 & $0.42-1.45$ & 0.43 \\
\hline Type of infection & & & \\
\hline Meningitis & 0.80 & $0.22-2.84$ & 0.72 \\
\hline SSI & 0.38 & $0.05-2.89$ & 0.35 \\
\hline Other & 1.42 & $0.77-2.63$ & 0.26 \\
\hline
\end{tabular}

negative cases, which can make treatment decisions very difficult, and the cases were $100 \%$ culture proven.

Acinetobacter spp. were isolated in $45 \%$ of cases, with Klebsiella spp. and $P$. aeruginosa the next most common isolates. The increase in the rate of Acinetobacter PCM has been extensively described in the years following 2000, although in the previous years it was reported as a rare occurrence. $4,5,18,22,28,32,36$ The main issue with the Acinetobacter spp. is their increasing resistance to almost all antibiotic classes (including carbapenems), which has been a significant problem in the recent years. . $^{1,4,10,16,22,25,30,36}$ Klebsiella spp. were the second most commonly isolated pathogens in this cohort, with the isolates carrying a high degree of resistance to third-generation cephalosporins and carbapenems (Table 4). One hundred percent of the isolates were sensitive to tetracycline, but during the study period the sensitivity to tigecycline was not routinely recorded.

The median CSF glucose/serum glucose ratio was 0.40 (IQR 0.40-0.47). Since all the cases were culturepositive, it is safe to assume that this rate is very suggestive of microbial meningitis as it has been reported previously. ${ }^{16}$ The $100 \%$ culture positivity rate is a rare occurrence in the literature. A positivity rate of $32 \%$ was reported in another prospective study. ${ }^{28}$ Retrospective studies by Kourbeti et al. have demonstrated a positivity rate ranging from $50 \%{ }^{14}$ to $74 \%$ (unpublished data). In those studies there were not any differences in the CSF characteristics between culture-positive and culture-negative cases. ${ }^{14}$ The mean duration from operation to the onset of symptoms was 6.5 days, an interval similar to that reported before. ${ }^{14,15,28}$

\section{Risk Factors}

The independent risk factors identified by the multivariate analysis were use of perioperative steroids, CSF leak, and the use of EVDs.

Craniotomies with an EVD carry a higher risk for meningitis. ${ }^{15,19,20,24,28}$ Ventriculitis in association with ventricular drain placement was a frequent problem and was observed in $11 \%$ of general neurosurgical patients in the series by Mayhall et al. ${ }^{20}$ There is little agreement among various series regarding the relation between duration of drainage and infection incidence; in one report, no relation was observed. ${ }^{19}$ Lozier et al. ${ }^{17}$ have commented on the controversy regarding the significance of the duration of EVD use with respect to the development of infections. At the University of Crete there were no routine changes of EVDs. Leaving an uninfected drain in place may be the best policy. ${ }^{15}$ In our institution, dressings were changed daily, the catheters were replaced at the first sign of infection or malfunction. Antibiotic-coated catheters were not routinely placed; we used them after the first catheter change for any reason or in patients who already had signs of infection and needed an EVD placed because of intraventricular hemorrhage or obstructive hydrocephalus.

Previous studies demonstrated postoperative CSF leak to be a significant risk factor for the development of meningitis..$^{2,11,12,23,26}$ In our series, the presence of CSF leak was associated with a 48 -fold increase in the risk of meningitis, and this was statistically significant. This is much higher than the risk we had identified in a previous retrospective study, ${ }^{15}$ in which the CSF leak increased the risk of meningitis by a factor of 1.7 but was not a statistically significant risk factor. Surgeons should be very vigilant in correcting a CSF leak. When a patient with a post-craniotomy CSF leak develops a fever, the possibility of meningitis should be strongly considered.

Meningitis or positive CSF culture has been described as the most frequent complication of intracranial pressure (ICP) monitoring. ${ }^{27}$ In this study, however, the presence of an ICP monitoring device did not affect the development of meningitis.

In contrast to findings of a previous prospective study, ${ }^{26}$ in our study duration of surgery did not remain an independent risk factor in the multivariate analysis. ${ }^{26}$ Age, sex, and diabetes were not significant risk factors for the development of meningitis, a finding consistent with previously reported data. ${ }^{12}$ Repeat surgery, which has been a major risk factor in recent ${ }^{28}$ and older ${ }^{26}$ reports, was not a risk factor in our cohort. Scheduling the procedure as an emergency or not an emergency was not a risk factor for PCM, a finding that we had described before. ${ }^{15}$ Entering a sinus was not a risk factor in this prospective study, in contrast to our retrospective report, ${ }^{15}$ and the reports of others. ${ }^{11,23}$

The use of perioperative steroids achieved a statistical significance in this prospective study. This has not been described before in studies regarding PCM and in most studies regarding postoperative wound infections in general neurosurgical patients. . $^{4,12-15,23,26,28,33}$ The effect of steroids on patients undergoing neurosurgical procedures has been variably reported. ${ }^{21}$ In certain studies, the steroid effect has not been specifically investigated.,12,13 Walcott et al. ${ }^{35}$ mention that for rare events such as infections in neurosurgery, it may be difficult to accomplish proof for the association of certain variables. In the study by Erman et al., ${ }^{5}$ perioperative steroid use did not remain an independent risk factor for the development of SSIs in the multivariate analysis. In the prospective study by Reichert et al. ${ }^{28}$ and the retrospective study by Kourbeti et al. ${ }^{15}$ on the risk factors associated with PCM, steroid administration did not reach a statistical significance. In studies in which steroids were administered to patients undergoing implantation of subdural electrodes, steroids had no effect on the incidence of infection. ${ }^{7,34}$ McPhee et al..$^{21}$ demonstrated a significant increase in postoperative wound infection and breakdown with steroid use in the setting of spinal operations for metastases, but they did not mention the effect on the development of meningitis. ${ }^{21}$ Shinoura et al. ${ }^{31}$ did not 
find that steroid use was a significant factor in a population of patients undergoing craniotomy for brain tumors. In summary, this is a matter that is highly controversial in the literature. Well-designed prospective studies will be needed in order to define the safe period for steroid use in neurosurgical patients.

\section{Microbiology of Other Infections}

Acinetobacter spp. were the predominant pathogens in VAP in this cohort (identified in $44 \%$ of cases), in accordance with the literature in critically ill patients., ${ }^{3,29}$ These pathogens are of extreme importance because they may be associated with a high rate of mortality $(78 \%$ in one series $^{29}$ ). The VAP isolates were $100 \%$ sensitive to colistin, like the PCM isolates, but their sensitivity to carbapenems and aminoglycosides was better than that of the PCM isolates.

Our study contains a tremendous amount of data on this prospective cohort of patients undergoing craniotomy. We believe that the prospective nature of the study and the sound statistical methods increase the power of our findings. Nevertheless, our population consisted mainly of patients with traumatic brain injury (50\%), so the overall severity of illness was high. Our results are sound, but still they do not allow us to make recommendations on the mitigation of the risk factors that predispose to meningitis. What we do suggest, however, is that in a patient who has received perioperative steroids, is being treated with a ventricular drain, has a CSF leak, or has a combination of these risk factors and has new-onset fever, meningitis should be considered. The fact that we detected culture-positive meningitis in $100 \%$ of the cases is probably due to the fact that several of the risk factors had been described before and we had an increased awareness for diagnosis of the infection in our cohort. This is very important especially since PCM often presents with subtle symptoms. ${ }^{14}$

Our results are largely confirmatory, and no new findings were demonstrated, except for the risk factor of the perioperative steroids, which has not been extensively discussed in the literature before. The description of the pathogens and their resistance profiles might be applicable to our academic center only. Despite this limitation, we believe that this extensive description of infections and pathogens may assist physicians to choose antibiotics that cover the most prevalent pathogens in patients presenting with fever after a craniotomy. Acinetobacter spp. remain very important pathogens with an increasing resistance to several antibiotic classes, a disturbing occurrence that affects patients' care all over the world. ${ }^{10}$ The finding that almost $40 \%$ of patients who underwent craniotomy in our prospective study developed at least one infection is disturbing, and it should alert physicians and investigators to search for the factors that will help in the mitigation of such complications in neurosurgical patients.

\section{Conclusions}

In this prospective study we were able to confirm the importance of perioperative ventricular drains and CSF leak in the development of PCM. We also demonstrated the significance of perioperative steroids in the development of meningitis. The duration of surgery was not a significant risk factor for PCM. As the first prospective surveillance of infections in patients undergoing craniotomy in Greece, this study is important because the offending pathogens and sensitivities for the most prevalent infections were described. We believe that this will greatly help physicians with the proper empiric choice of antibiotics in this population.

\section{References}

1. Briggs S, Ellis-Pegler R, Raymond N, Thomas M, Wilkinson L: Gram-negative bacillary meningitis after cranial surgery or trauma in adults. Scand J Infect Dis 36:165-173, 2004

2. Blomstedt GC: Craniotomy infections. Neurosurg Clin N Am 3:375-385, 1992

3. Chastre J, Fagon JY: Ventilator-associated pneumonia. Am J Respir Crit Care Med 165:867-903, 2002

4. Erdem I, Hakan T, Ceran N, Metin F, Akcay SS, Kucukercan M, et al: Clinical features, laboratory data, management and the risk factors that affect the mortality in patients with postoperative meningitis. Neurol India 56:433-437, 2008

5. Erman T, Demirhindi H, Göçer AI, Tuna M, Ildan F, Boyar B: Risk factors for surgical site infections in neurosurgery patients with antibiotic prophylaxis. Surg Neurol 63:107113,2005

6. Gulati S, Kapil A, Das B, Dwivedi SN, Mahapatra AK: Nosocomial infections due to Acinetobacter baumannii in a neurosurgery ICU. Neurol India 49:134-137, 2001

7. Hersh EH, Virk MS, Shao H, Tsiouris AJ, Bonci GA, Schwartz TH: Bone flap explantation, steroid use, and rates of infection in patients with epilepsy undergoing craniotomy for implantation of subdural electrodes. Clinical article. J Neurosurg 119:48-53, 2013

8. Horan TC, Andrus M, Dudeck MA: CDC/NHSN surveillance definition of health care-associated infection and criteria for specific types of infections in the acute care setting. Am J Infect Control 36:309-332, 2008

9. Hsu GJ, Young TG, Chou JW, Peng MY, Chang FY, Chou MY: Gram-negative bacillary meningitis in adults. J Formos Med Assoc 92:317-323, 1993

10. Kim BN, Peleg AY, Lodise TP, Lipman J, Li J, Nation R, et al: Management of meningitis due to antibiotic-resistant Acinetobacter species. Lancet Infect Dis 9:245-255, 2009

11. Korinek AM: [Prevention of meningitis after craniotomy in scheduled surgery.] Ann Fr Anesth Reanim 11:711-715, $1992(\mathrm{Fr})$

12. Korinek AM, French Study Group of Neurosurgical Infections, SEHP, C-CLIN Paris-Nord: Risk factors for neurosurgical site infections after craniotomy: a prospective multicenter study of 2944 patients. Neurosurgery 41:1073-1081, 1997

13. Korinek AM, Golmard JL, Elcheick A, Bismuth R, van Effenterre R, Coriat P, et al: Risk factors for neurosurgical site infections after craniotomy: a critical reappraisal of antibiotic prophylaxis on 4,578 patients. Br J Neurosurg 19:155-162, 2005

14. Kourbeti IS, Jacobs AV, Koslow M, Karabetsos D, Holzman RS: Risk factors associated with postcraniotomy meningitis. Neurosurgery 60:317-326, 2007

15. Kourbeti IS, Vakis AF, Papadakis JA, Karabetsos DA, Bertsias G, Filippou M, et al: Infections in traumatic brain injury patients. Clin Microbiol Infect 18:359-364, 2012

16. Leib SL, Boscacci R, Gratzl O, Zimmerli W: Predictive value of cerebrospinal fluid (CSF) lactate level versus CSF/blood glucose ratio for the diagnosis of bacterial meningitis following neurosurgery. Clin Infect Dis 29:69-74, 1999 
17. Lozier AP, Sciacca RR, Romagnoli MF, Connolly ES Jr: Ventriculostomy-related infections: a critical review of the literature. Neurosurgery 51:170-182, 2002

18. Lu CH, Chang WN, Chuang YC, Chang HW: Gram-negative bacillary meningitis in adult post-neurosurgical patients. Surg Neurol 52:438-444, 1999

19. Mahé V, Kermarrec N, Ecoffey C: [Infections related to external ventricular drainage.] Ann Fr Anesth Reanim 14:8-12, 1995 (Fr)

20. Mayhall CG, Archer NH, Lamb VA, Spadora AC, Baggett JW, Ward JD, et al: Ventriculostomy-related infections. A prospective epidemiologic study. N Engl J Med 310:553 559,1984

21. McPhee IB, Williams RP, Swanson CE: Factors influencing wound healing after surgery for metastatic disease of the spine. Spine (Phila Pa 1976) 23:726-733, 1998

22. Metan G, Alp E, Aygen B, Sumerkan B: Carbapenem-resistant Acinetobacter baumannii: an emerging threat for patients with post-neurosurgical meningitis. Int $\mathbf{J}$ Antimicrob Agents 29:112-113, 2007

23. Mollman HD, Haines SJ: Risk factors for postoperative neurosurgical wound infection. A case-control study. J Neurosurg 64:902-906, 1986

24. Narotam PK, van Dellen JR, du Trevou MD, Gouws E: Operative sepsis in neurosurgery: a method of classifying surgical cases. Neurosurgery 34:409-416, 1994

25. Nguyen MH, Harris SP, Muder RR, Pasculle AW: Antibioticresistant Acinetobacter meningitis in neurosurgical patients. Neurosurgery 35:851-855, 1994

26. Patir R, Mahapatra AK, Banerji AK: Risk factors in postoperative neurosurgical infection. A prospective study. Acta Neurochir (Wien) 119:80-84, 1992

27. Rebuck JA, Murry KR, Rhoney DH, Michael DB, Coplin WM: Infection related to intracranial pressure monitors in adults: analysis of risk factors and antibiotic prophylaxis. J Neurol Neurosurg Psychiatry 69:381-384, 2000

28. Reichert MCF, Medeiros EAS, Ferraz FAP: Hospitalacquired meningitis in patients undergoing craniotomy: incidence, evolution, and risk factors. Am J Infect Control 30:158-164, 2002

29. Rello J: Acinetobacter baumannii infections in the ICU: customization is the key. Chest 115:1226-1229, 1999

30. Rodriguez CH, Bombicino K, Granados G, Nastro M, Vay C, Famiglietti A: Selection of colistin-resistant Acinetobacter baumannii isolates in postneurosurgical meningitis in an intensive care unit with high presence of heteroresistance to colistin. Diagn Microbiol Infect Dis 65:188-191, 2009 (Erratum in Diagn Microbiol Infect Dis 66:341, 2009)

31. Shinoura N, Yamada R, Okamoto K, Nakamura O: Early prediction of infection after craniotomy for brain tumours.

Br J Neurosurg 18:598-603, 2004

32. Siegman-Igra Y, Bar-Yosef S, Gorea A, Avram J: Nosocomial acinetobacter meningitis secondary to invasive procedures: report of 25 cases and review. Clin Infect Dis 17:843-849, 1993

33. Srinivas D, Veena Kumari HB, Somanna S, Bhagavatula I, Anandappa CB: The incidence of postoperative meningitis in neurosurgery: an institutional experience. Neurol India 59:195-198, 2011

34. Van Gompel JJ, Worrell GA, Bell ML, Patrick TA, Cascino GD, Raffel C, et al: Intracranial electroencephalography with subdural grid electrodes: techniques, complications, and outcomes. Neurosurgery 63:498-506, 2008

35. Walcott BP, Redjal N, Coumans JVCE: Infection following operations on the central nervous system: deconstructing the myth of the sterile field. Neurosurg Focus 33(5):E8, 2012

36. Wroblewska MM, Dijkshoorn L, Marchel H, van den Barselaar M, Swoboda-Kopec E, van den Broek PJ, et al: Outbreak of nosocomial meningitis caused by Acinetobacter baumannii in neurosurgical patients. J Hosp Infect 57:300-307, 2004

\section{Author Contributions}

Conception and design: Kourbeti, Vakis, Ziakas, Samonis. Acquisition of data: Kourbeti, Vakis, Karabetsos, Potolidis, Christou. Analysis and interpretation of data: Ziakas. Drafting the article: Kourbeti, Vakis, Ziakas, Karabetsos, Potolidis, Samonis. Critically revising the article: Kourbeti, Vakis, Samonis.

Reviewed submitted version of manuscript: Kourbeti, Vakis, Karabetsos, Potolidis, Christou, Samonis. Approved the final version of the manuscript on behalf of all authors: Kourbeti. Statistical analysis: Ziakas. Administrative/technical/material support: Vakis, Samonis. Study supervision: Kourbeti, Vakis, Ziakas, Karabetsos, Samonis

\section{Supplemental Information \\ Previous Presentation}

Portions of this work were presented at the 22nd European Congress of Clinical Microbiology and Infectious Diseases (ECCMID), London, March 31-April 3, 2012.

\section{Correspondence}

Irene S. Kourbeti, Department of Acute and Emergency Medicine, Furness General Hospital, Dalton Ln., Barrow in Furness, Cumbria LA14 4LF, United Kingdom. email: irenekourbeti@ hotmail.com. 